Bull. Mater. Sci., Vol. 23, No. 4, August 2000, pp. 267-272. (C) Indian Academy of Sciences.

\title{
Specific heat studies in Ho-Ba-CuO superconductors: Fermionic and bosonic contributions
}

\author{
DINESH VARSHNEY*, SANJAY SHAH and R K SINGH ${ }^{\dagger}$ \\ School of Physics, Devi Ahilya University, Khandwa Road Campus, Indore 452 017, India \\ ${ }^{\dagger}$ School of Pure and Applied Physics, Guru Ghasidas University, Bilaspur 495 009, India
}

MS received 11 November 1999

\begin{abstract}
The specific heats of superconducting $\mathrm{HoBa}_{2} \mathrm{Cu}_{3} \mathrm{O}_{7-\delta}\left(T_{\mathrm{c}} \cong 92 \mathrm{~K}\right)$ have been theoretically investigated in the temperature domain $70 \leq T \leq 110 \mathrm{~K}$. The bosonic (phonons) contribution to the specific heat is estimated from Debye model in the harmonic approximation for high temperature expansion $\left(T>\theta_{\mathrm{D}} / 2 \pi\right) \mathrm{using}$ the moments of the phonon density of states. The fermionic constituent as the electronic specific heat is deduced using a suitable trial function above and below $T_{\mathrm{c}}$. As a next step the contribution of specific heat by charge oscillations (plasmons) are obtained. The theoretical results from bosonic and fermionic terms are then compared with the experimental results. We find that the specific heats from electronic as well as plasmon term are only a fraction of lattice specific heat and in particular, plasmons do not influence the thermal conduction significantly. The implications of the above analysis are discussed.
\end{abstract}

Keywords. Electronic; phonons; plasmons; specific heat; Ho-based superconductors.

\section{Introduction}

The remarkable achievement of high- $T_{\mathrm{c}}$ superconductivity in cuprates, $\mathrm{La}_{2-x} \mathrm{Sr}_{x} \mathrm{CuO}_{4}$ (Bednorz and Muller 1986) and $\mathrm{YBa}_{2} \mathrm{Cu}_{3} \mathrm{O}_{7-\delta}$ (Wu et al 1987) has attracted considerable attention of experimental and theoretical workers during the past one decade. In the course of discovery, much attention has been focused on the carrier dynamics along as well as perpendicular to the superconductor copper oxide plane. From the point of view of structure the cuprates have in general a common feature of $\mathrm{CuO}_{2}$ plane. The copper charge neutralities are perturbed either by doping concentration $(x)$ [e.g. in $\mathrm{La}_{2-x} \mathrm{Sr}_{x} \mathrm{CuO}_{4}$ ] or by oxygen deficiency $(\delta)$ [as in $\mathrm{YBa}_{2} \mathrm{Cu}_{3} \mathrm{O}_{7-\delta}$. The salient features of high- $T_{\mathrm{c}}$ cuprates are (i) charge carriers are confined to two-dimensional (2-D) network, (ii) a nearly linear behaviour of normal state resistivity above $T_{\mathrm{c}}$, (iii) a reduced oxygen isotope effect on $T_{\mathrm{c}}$, (iv) a kink in the behaviour of specific heat in the vicinity of $T_{\mathrm{c}}$ and (v) a $d$-wave symmetry, $d_{x^{2}-y^{2}}$ pairing state. These observations point towards the possible explanation of nature of the pairing mechanism that leads to strongly bound pairs in the superconducting state. To account such characteristics by phonon mechanism is one of the most captivating debates in high- $T_{\mathrm{c}}$ cuprates.

There exists a great body of experimental data on the thermal properties in particular, specific heat study has fascinated as it is used as a tool to understand the bulk

*Author for correspondence properties of these materials both at very low temperature $(T \sim 10 \mathrm{~K})$ and near the transition temperature. At low temperature, a linear term $(\gamma T)$ and in the vicinity of $T_{\mathrm{c}}$ a discontinuity is observed in the temperature dependence of specific heat in most of the cuprate superconductors (Junod 1990). It is worth mentioning that in normal metal at low temperatures, electrons near the Fermi surface contribute to a linear term $\gamma^{\prime} T$ to the specific heat. At higher temperatures, this contribution becomes difficult to predict because of the dominant contribution by phonons. According to BCS theory (Bardeen et al 1957), below $T_{\mathrm{c}}$, an energy gap of $2 \Delta$ forms at Fermi surfaces for conventional superconductors. For low temperatures i.e. $T \ll \Delta / k_{\mathrm{B}}$, the energy gap prevents the normal electrons to contribute significantly to specific heat. BCS theory predicts electronic contribution to the low temperature specific heat through the expression

$$
C=A \exp \left[-\Delta / k_{\mathrm{B}}\right] .
$$

Switching to high- $T_{\mathrm{c}}$ cuprates, in particular $\mathrm{HoBa}_{2} \mathrm{Cu}_{3} \mathrm{O}_{7-\delta}$ $\left(T_{\mathrm{c}} \cong 92 \mathrm{~K}\right)$ is a homologue to $\mathrm{YBa}_{2} \mathrm{Cu}_{3} \mathrm{O}_{7-\delta}$, has complicated electronic structure. The layered structure consists of superconducting planes and the transport properties of YBCO based systems are strongly dependent on the oxygen deficiency $(\delta)$. It may be noted that for the lower values of $\delta(\cong 0 \cdot 0)$ the phase is orthorhombic and becomes superconducting at low temperatures. With the increase in oxygen deficiency $\delta(\cong 1 \cdot 0)$, the phase is tetragonal and non-superconducting at all temperatures. As regards 
the behaviour of $T_{\mathrm{c}}$ with oxygen deficiency is concerned, it is anomalous which has been noticed since its discovery. The orthorhombic phase of $\mathrm{HoBa}_{2} \mathrm{Cu}_{3} \mathrm{O}_{7-\delta}$ is composed of two-dimensional (2-D) $\mathrm{CuO}_{2}$ layers as well as one-dimensional (1-D) $\mathrm{CuO}$ chains. It is believed that both 2-D planes and 1-D chains are metallic in nature.

The intriguing features of the reported specific heat measurements on cuprates includes a linear term $\left(\gamma^{\prime} T\right)$ at very low temperatures, an upturn in $C(T)$ of superconducting version in the vicinity of $T_{\mathrm{c}}$ and a bump in the normal state region near to room temperature. The low temperature specific heat measurements have been reported by Ferreira et al (1998) in the temperature domain $(0.5$ and $30-50 \mathrm{~K})$. The principal finding of the measurement is at low temperatures and suggests the presence of Schottky type anomalies arising from the interaction of rare earth ions with crystalline electric field. It is emphasized that the specific heat below $T_{\mathrm{c}}$ can be resolved into a $\gamma^{\prime} T$ contribution with a finite value of $\gamma^{\prime}$ and a lattice contribution. Later on, Naumov et al (1996) measured the heat capacity of $\mathrm{HoBa}_{2} \mathrm{Cu}_{3} \mathrm{O}_{7-\delta}$ superconductor in the temperature range $14 \leq T \leq 315 \mathrm{~K}$. The contribution to electronic term $\left(\gamma^{\prime} T\right)$ was extracted from the total heat capacity in the region of normal state and argue that this contribution is mainly due to the electronic heat capacity.

The observation of the anomalous behaviour has, therefore, been of considerable interest. It seems essential to point out that these anomalies represent a deviation from the BCS prediction. High resolution electron microscopy techniques have been used to probe the electronic structure of cuprate superconductors. Electron energy loss spectroscopy (EELS) reveals a free carrier plasmon at an energy of about 1-2 eV (Bozovic 1990). This indicates that charge fluctuations in cuprates are large in the cuprates and may contribute significantly to the pairing mechanism. Earlier, Kresin et al (1993) emphasized the role of plasmons in the pairing mechanism as well as in yielding a higher value of $T_{c}$. Recently, Leggett (1999) have shown the plausible explanation of the systematic of $T_{\mathrm{c}}$ in the layered structure with charge oscillations (plasmons). It is therefore of importance to see the possible role of plasmons in the thermal transport of high- $T_{\mathrm{c}}$ cuprates.

The investigations are organized as follows. In $\S 2$, we aim to derive the electronic, phononic and plasmonic contribution to specific heat for $\mathrm{HoBa}_{2} \mathrm{Cu}_{3} \mathrm{O}_{7-\delta}$ superconductors. The reason for selection of Ho123 system is the availability of experimental information. Section 3 is devoted to results and discussion. Looking at the importance of charge oscillations (plasmons) in the pairing mechanism, we made a relative comparison of these contributions and finally the total contribution to specific heat was then compared with the one obtained from the experiments. We conclude in $\$ 4$.

\section{The model}

The single crystal of $\mathrm{HoBa}_{2} \mathrm{Cu}_{3} \mathrm{O}_{7-\delta}$ superconductors can be modelled to an infinite array of two-dimensional (2-D) $\mathrm{CuO}_{2}$ layers as well as one-dimensional (1-D) $\mathrm{CuO}$ chains. The layered structure is regarded as stack of planes of $\mathrm{Ho}, \mathrm{CuO}_{2}, \mathrm{BaO}, \mathrm{CuO}$ (chains), $\mathrm{BaO}, \mathrm{CuO}_{2}$, Ho, .... We consider a two-dimensional electron gas where the charge carriers interact by way of polarized waves as quantized lattice vibrations (phonons) and charge oscillations (plasmons) in the conducting $\mathrm{CuO}_{2}$ plane. With the removal of oxygen content $(\delta)$ in $\mathrm{HoBa}_{2} \mathrm{Cu}_{3} \mathrm{O}_{7-\delta}$ superconductor, sufficient number of free carriers (holes) will develop in the $\mathrm{CuO}_{2}$ plane and participate in the conduction mechanism. The holes as carriers form a pair via phonons as well as plasmons in $\mathrm{CuO}_{2}$ plane. We stress that the concentration of holes as carriers (for $p$-type $\mathrm{HoBa}_{2} \mathrm{Cu}_{3} \mathrm{O}_{7-\delta}$ ) in these planes is one of the most universal parameters which affects both the normal and superconducting state properties of cuprates.

\subsection{Electronic specific heat}

Let us start with the estimation of the electronic specific heat in the normal state of $\mathrm{Ho}-\mathrm{Ba}-\mathrm{CuO}$ where the holes as carriers are within the spherical Fermi surface. The internal energy of a normal metal is expressed as

$$
U=U_{0}+\frac{m^{*} k_{\mathrm{F}}}{6 \hbar^{2}}\left(k_{\mathrm{B}} T\right)^{2},
$$

with $m^{*}$ the effective mass of the holes as carriers and $k_{\mathrm{F}}$ denotes Fermi wave vector. The temperature derivative of the internal energy yields the specific heat and is

$$
C_{\mathrm{el}}=\frac{m^{*} k_{\mathrm{F}} k_{\mathrm{B}}^{2}}{3 \hbar^{2}} T=\gamma T .
$$

It is evident from (2) that the electronic specific heat is influenced by (i) the effective mass of the carriers and (ii) the carrier concentration. Most of the specific heat measurements show a discontinuity in $C(T)$ of superconducting version in the vicinity of transition temperature. In order to have better perception of the occurrence of an anomaly at $T \sim T_{\mathrm{c}}$ of electronic specific heat, we use a trial function in the two-temperature domain as follows

$$
\begin{aligned}
C_{\text {ele }} & =\gamma T & & \text { for } T>T_{\mathrm{c}} \\
& =\frac{3 \gamma T^{3}}{T_{\mathrm{c}}^{2}} & & \text { for } T<T_{\mathrm{c}} .
\end{aligned}
$$

In the vicinity of $T_{\mathrm{c}}$, the specific heat is dominated by lattice contribution and we now estimate the lattice specific heat in the following subsection. 


\subsection{Lattice specific heat}

In most of high- $T_{\mathrm{c}}$ cuprates, the specific heat is dominated by quantized lattice vibrations (phonons) and is well estimated by the Debye theory. As phonons are bosons and waves of all frequencies in the range $0<\omega<\omega_{\mathrm{m}}$ could propagate through crystal. The internal energy of the crystal when the maximum energy of the phonons in the crystal is $\xi=\hbar \omega$ (Ghatak and Kothari 1972) is

$$
U=U_{0}+\frac{9 N}{\left(k_{\mathrm{B}} \theta_{\mathrm{D}}\right)^{3}} \int_{0}^{k_{\mathrm{B}} \theta_{\mathrm{D}}} \frac{\xi^{3} \mathrm{~d} \xi}{e^{\xi / k_{\mathrm{B}} T}-1},
$$

with $\theta_{\mathrm{D}}$ as the Debye temperature and $N$ the number of atoms in a unit cell. The specific heat from lattice contribution is obtained by temperature derivative as

$$
C_{\text {latt }}=\frac{9 N}{\left(k_{\mathrm{B}} \theta_{\mathrm{D}}\right)^{3}} \int_{0}^{k_{\mathrm{B}} \theta_{\mathrm{D}}} \frac{\xi^{4} e^{\xi / k_{\mathrm{B}} T}}{k_{\mathrm{B}} T^{2}\left(e^{\xi / K T}-1\right)^{2}} \mathrm{~d} \xi .
$$

Also,

$$
C_{\text {latt }}=3 N k_{\mathrm{B}} f_{\mathrm{D}}\left(\theta_{\mathrm{D}}, T\right),
$$

with

$$
f_{\mathrm{D}}\left(\theta_{\mathrm{D}}, T\right)=\frac{1}{x_{\mathrm{m}}^{3}}\left[12 \int_{0}^{x_{\mathrm{m}}} \frac{x^{3} \mathrm{~d} x}{e^{x}-1}-\frac{3 x_{\mathrm{m}}^{4}}{e^{x_{\mathrm{m}}}-1}\right],
$$

and $x_{\mathrm{m}}=\theta_{\mathrm{D}} / T$.

The typical Debye temperature in cuprates is $400 \mathrm{~K}$ and hence in high temperature limit i.e. $T>\left(\theta_{\mathrm{D}} / 2 \pi\right)$, the expansion of (6) within harmonic approximation yields

$$
C_{\text {latt }}=3 N k_{\mathrm{B}}\left[1-\frac{\theta_{2}^{2}}{12 T^{2}}-\frac{\theta_{4}^{4}}{\theta_{*}^{4}}+\frac{\theta_{4}^{4}}{12 T^{2} \theta_{*}^{2}}\right] .
$$

The moments of the phonon density of states are obtained from real phonon spectrum as, the second moment $\theta_{2}$, the fourth moment $\theta_{4}$, and the moment attribute to upper limit $\theta_{*}$, of the phonon spectrum. As a next step we estimate the plasmonic specific heat, although in pure BCS case no plasmonic specific heat exists.

\subsection{Plasmonic specific heat}

The present state of theory for attractive pairing mechanism in high- $T_{\mathrm{c}}$ cuprates suggests that charge oscillations (plasmons) plays an apex role because of layer stacking sequence. The physical explanation is as follows: an important fact is the dispersion relation for the charge oscillations in long wavelength limit

$$
\omega(q)=a q+b q^{1 / 2},
$$

with $a$ being $v_{\mathrm{F}} / \sqrt{2}$ and $b$ the plasmon velocity. It appears that in an interacting many particles system, which is described by high-energy charge oscillations, it will contribute to the conduction, i.e. specific heat in the temperature domain where they become thermally mobile. This temperature dependent contribution is influenced by the change in oxygen content. The tunnelling of collective mode of charged particle into the particle hole spectrum of the metal is known in high- $T_{\mathrm{c}}$ superconductors. It has been, therefore, of considerable interest to show their role in specific heat.

The plasmon contribution to internal energy is in the superfluid state and associated with the plasmon velocity, $V_{\mathrm{pl}}(T)$. One can write the internal energy as

$$
U_{\mathrm{pl}}=U_{\mathrm{pl}}(0)+\frac{\pi^{2} N\left(k_{\mathrm{B}} T\right)^{4}}{30\left[\hbar V_{\mathrm{pl}}(T)\right]^{3}} .
$$

The specific heat from this contribution is obtained by temperature derivative as

$$
C_{\mathrm{pl}}(T)=\frac{\pi^{2} N\left(k_{\mathrm{B}} T\right)^{4}}{30\left[\hbar V_{\mathrm{pl}}(T)\right]^{3}}\left[\frac{4}{T}-\frac{3}{V_{\mathrm{pl}}} \frac{\mathrm{d} V_{\mathrm{pl}}}{\mathrm{d} T}\right],
$$

assuming that $V_{\mathrm{pl}}(T)$ does not change much in temperature domain $70 \leq T \leq 110 \mathrm{~K}$, and hence,

$$
C_{\mathrm{pl}}(T)=\frac{4 \pi^{2} N k_{\mathrm{B}}^{4} T^{3}}{30\left[\hbar V_{\mathrm{pl}}(T)\right]^{3}} .
$$

With the above description the total contribution to specific heat is

$$
C_{\text {total }}=C_{\text {elec }}+C_{\text {lattice }}+C_{\mathrm{pl}} \text {. }
$$

Using the developed expression for bosonic and fermionic specific heat, we have estimated the specific heat in $\mathrm{HoBa}_{2} \mathrm{Cu}_{3} \mathrm{O}_{7-\delta}$ superconductors. The calculated results along with discussion are presented in the following section.

\section{Results and discussion}

It is interesting to discuss first the possible difficulties in explaining the observed anomalous behaviour. As far as a linear term in the low temperature domain, it is unclear that $\gamma^{\prime} T$ is associated with non-superconducting phase or is an intrinsic property due to vanishing of energy gap over a portion of Fermi surface. In the vicinity of $T_{\mathrm{c}}$, the 
lattice contribution to specific heat is dominating, so separation of fermionic term from the phononic effect is difficult without an accurate model for phononic specific heat. Such a model requires many unknown input parameters. Besides these, there is a possibility of having other phase transitions, either electronic or lattice near to the room temperature. In the present situation, it is worthwhile to explore efforts to understand the observed discontinuity near $T_{\mathrm{c}}$.

We have plotted the theoretical results on electronic specific heat of $\mathrm{HoBa}_{2} \mathrm{Cu}_{3} \mathrm{O}_{7-\delta}$ superconductors with temperature for the domain $70 \leq T \leq 110 \mathrm{~K}$ from (3) developed in $\$ 2$ and shown in figure 1 . The Sommerfield constant is used as $35 \mathrm{~mJ} / \mathrm{mol} / \mathrm{K}^{2}$ for $\mathrm{HoBa}_{2} \mathrm{Cu}_{3} \mathrm{O}_{7-\delta}$ with $T_{\mathrm{c}}=92.3 \mathrm{~K}$ (Tarascan et al 1987). For temperature $<T_{\mathrm{c}}$, a correct representation of specific heat is complicated and hence we have used this trial form. In the temperature domain $T>T_{\mathrm{c}}$, the electron specific heat contributes linearly. The above form of $C_{\text {ele }}$ allows having a sharp decrease at $T=T_{\mathrm{c}}$, which is reported by a number of experimental groups (Junod 1990). We find that the above trial form along with $\gamma$ values provides a sharp discontinuity in the vicinity of $T_{\mathrm{c}}$.

The key feature of specific heat in high- $T_{\mathrm{c}}$ cuprate superconductors is the lattice contribution to specific heat and is well estimated from Debye model in the high temperature limit within harmonic approximation. The moments of density of states are directly obtained from the phonon

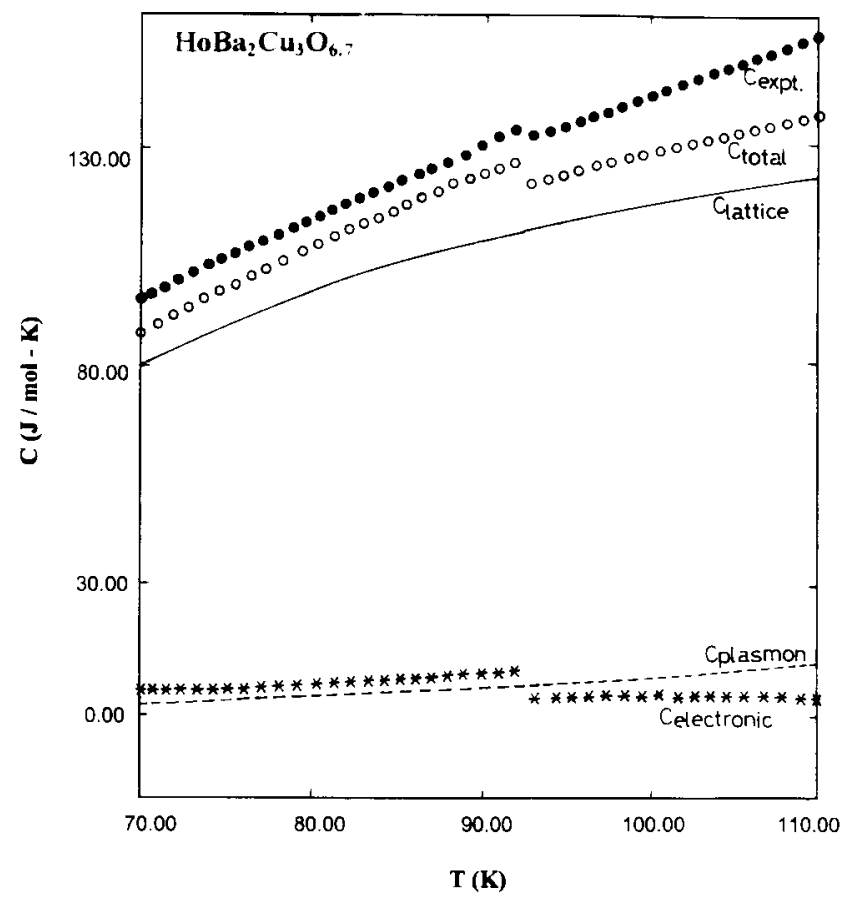

Figure 1. Variation of $C(\mathrm{~J} / \mathrm{mol}-\mathrm{K})$ with temperature in the range $70 \leq T \leq 110 \mathrm{~K}$. Closed circles are the data taken from Naumov et al (1996). spectrum of the system under consideration. This technique is rather precise as experimental information is directly used rather than estimating Debye temperature. As the numbers of atoms are 12.87 in unit cell, so a number of phonon branches exist and to estimate $\theta_{\mathrm{D}}$ theoretically is not precise. Furthermore, (8) is valid in high temperature limit, i.e. $T>\left(\theta_{\mathrm{D}} / 2 \pi\right)$. The phonon spectrum reveals the moments of the phonon density of states as $\theta_{2}=430 \mathrm{~K}, \theta_{4}=486 \mathrm{~K}$ and $\theta_{*}=570 \mathrm{~K}$ (Atake et al 1989; Naumov et al 1996) which are used to estimate the lattice specific heat. The results on $C_{\text {latt }}$ with temperature is plotted in figure 1 . This power series in specific heat permits the harmonic lattice term to be fitted reasonably well down to $50 \mathrm{~K}$.

In the case of high- $T_{\mathrm{c}}$ cuprates, the contribution to specific heat by electronic term is well established besides bosonic (phonons) term. We anticipate that one of the possible contributions may arise from charge oscillations (plasmons) although in pure BCS case, clearly no plasmonic specific heat exists. The idea is based on the concept that the plasmons contribute significantly to the pairing mechanism and transition temperature. It seems essential to seek their contribution to transport properties, as the plasmons are thermally mobile in temperature range of interest although there are no experimental evidences for their contribution to specific heat.

Let us now discuss the results for the plasmonic contribution to specific heat. The plasmon velocity is estimated from the relation

$$
V_{\mathrm{pl}}=a k_{\mathrm{B}} \omega_{\mathrm{p}} /\left(\hbar^{3} \pi^{3}\right),
$$

with $\omega_{\mathrm{p}}(=1 \mathrm{eV})$ as the plasma frequency (Bozovic 1990) and $a(=3.8 \AA)$ the lattice parameter (Tarascan et al 1987) to obtain $V_{\mathrm{pl}}=1.9 \times 10^{6} \mathrm{~cm} \mathrm{sec}^{-1}$. In principle, the plasmons are high-energy particles and are higher in energy by at least three orders of magnitude than phonons. Also, in conventional BCS theory as well as heat capacity measurements on high- $T_{\mathrm{c}}$ cuprates, no plasmonic heat is specified, but we find that in the superfluid state, the plasmons contribute to the specific heat although in very small way. It is noticed from (11) that $C_{\mathrm{pl}}$ infers a cubic law of temperature which is well evident from the plot. While estimating the plasmonic contribution, we assumed that the plasmon velocity does not change much in temperature region of interest. Precisely, the comparison of $C_{\text {ele }}$ and $C_{\mathrm{pl}}$ for high- $T_{\mathrm{c}}$ cuprates suggests that their contribution to specific heat is significantly small.

We aim to discuss both bosonic (phonon and plasmon) and fermionic (electronic) contribution to specific heat of high- $T_{\mathrm{c}} \mathrm{HoBa}_{2} \mathrm{Cu}_{3} \mathrm{O}_{7-\delta}$ superconductors in the temperature domain $70 \leq T \leq 110 \mathrm{~K}$. In figure 1 we compare the theoretical results with experimental data (Naumov et al 1996). By comparing different contributions to specific heat, one cannot escape by concluding that the electronic as well as plasmonic contributions are only a fraction of lattice 
specific heat. The major contribution to specific heat is from the quantized lattice vibrations (phonons) and is well estimated in the harmonic approximation for high temperature expansion by suitably using the moments of the phonon density of states. Below $T_{\mathrm{c}}, C$ increases and at $T \cong T_{\mathrm{c}}$, there is discontinuity because of electronic specific heat and $C$ further increases monotonously for $T>T_{\mathrm{c}}$.

The results obtained from the model calculations partially supports the inclusion of thermally mobile plasmons i.e. the plasmonic term, which in principle does not exist in the BCS theory as well as other models on high- $T_{\mathrm{c}}$ superconductors. A clear difference in between the theoretical plot and experimental points below $T_{\mathrm{c}}$ is thus obtained. At $T_{\mathrm{c}}$, the difference $\Delta C\left(T_{\mathrm{c}}\right) / T_{\mathrm{c}}$ is $0.08 \mathrm{~J} / \mathrm{mol} / \mathrm{K}^{2}$, together with electronic specific heat coefficient in the superconducting state. The ratio $\Delta C\left(T_{\mathrm{c}}\right) / \gamma T_{\mathrm{c}}$ is deduced as 2.25 which is higher than the BCS ratio of 1.43 in weak coupling theory. It was shown earlier by Carbotte (1990) that the ratio is about 2.8 using the joint phonon-exciton model. This clearly demonstrates the participation of both phonons and plasmons in the transport mechanism. We believe that the optimized doping in the parent compound produces localized holes, which is a key reason for the nonzero values of electronic specific heat coefficient at $T_{\mathrm{c}}$. Also the upturn in $C$ at $T_{\mathrm{c}}$ reduces the $\gamma$ value and hence the weak densities of states at Fermi level results.

\section{Conclusion}

In the present theoretical investigations, we have analysed the anomalous behaviour of specific heat of $\mathrm{HoBa}_{2} \mathrm{Cu}_{3} \mathrm{O}_{7-\delta}$ superconductors in the temperature domain $70 \leq T \leq 110 \mathrm{~K}$. We consider the conducting $\mathrm{CuO}_{2}$ plane as isolated and the electron gas lies in this plane. The charge carriers interact by way of the quantized lattice vibrations (phonons) as well as the charge oscillations (plasmons). We have critically analysed the contributions for the specific heat from (i) electronic, (ii) phononic, and (iii) plasmonic terms.

By assuming that there is definitely a major contribution from phonons, the lattice specific heat is well estimated in the framework of harmonic approximation for high temperature limit i.e. $T>\theta_{\mathrm{D}} / 2 \pi$. For this purpose we have properly incorporated the moments of the phonon density of states to predict the temperature dependence of lattice specific heat in $\mathrm{HoBa}_{2} \mathrm{Cu}_{3} \mathrm{O}_{7-\delta}$ superconductors. The aim of the present investigations is not to reveal anything particularly new but to draw attention on the independent and relative contributions from electronic, phononic as well as plasmonic terms.

We have used a suitable trial function for the estimation of electronic specific heat in the two-fluid model. It is noticed that the above form will provide a better perception of the electronic contribution in the vicinity of transi- tion temperature. Perhaps the dilemma in determining the electronic specific heat of a high- $T_{\mathrm{c}}$ superconductor is that it represents at best only a few percent of total specific heat, in particular near the vicinity of $T_{\mathrm{c}}$. Hence the separation of electronic specific heat from the lattice contribution is not an easy task.

The cuprates show the existence of plasmons because of layered stacking sequence and have a pronounced effect on the physical properties describing the superconducting state. In order to test their possible contribution in the thermal properties, we have made efforts to explore their role by estimating the plasmon velocity from the dispersion relation. The plasmons are thermally mobile in the temperature range of interest and we find that their contribution does exist although the magnitude is too small and comparable with the electronic contribution. From the point of view of BCS theory plasmonic contribution towards the specific heat does not occur, so it is believed that their contribution towards the transport mechanism will open a new understanding. In continuation, till to date there are no experimental evidences for the participation of plasmons towards specific heat.

In conclusion, a satisfactory agreement between the theoretically calculated and experimental data on the specific heat behaviour of $\mathrm{Ho}-\mathrm{Ba}-\mathrm{CuO}$ cuprate superconductors is obtained. We assumed that the plasmons in the temperature domain $70 \leq T \leq 110 \mathrm{~K}$ are thermally mobile in the $\mathrm{CuO}_{2}$ plane for the purpose of heat transport. The results for plasmonic and electronic specific heat are no doubt important for the observed behaviour, but we cannot escape in concluding that the lattice specific heat is a major contribution.

\section{Acknowledgements}

Financial assistance from the University Grants Commission, New Delhi, and UGC-Central Regional Office, Bhopal, are gratefully acknowledged.

\section{References}

Atake T, Zhang Q Z, Takagi Y and Saito Y 1989 Report of the research laboratory of engineering materials, Tokyo University, Tokyo Institute of Technology, 11

Bardeen J, Cooper L N and Schrieffer J R 1957 Phys. Rev. 108 1175

Bednorz J G and Muller K A 1986 Z. Phys. B64 189

Bozobic I 1990 Phys. Rev. B36 226

Carbotte J P 1990 Rev. Mod. Phys. 62207

Ferreira J M, Lee B W, Dalichaouch Y, Torikachvili M S, Yang K N and Maple M B 1988 Phys. Rev. B37 1480

Ghatak A K and Kothari L S 1972 An introduction to lattice dynamics (London: Addison Wiley Publ. Co.) 
Junod A 1990 in Physical properties of high temperature superconductors (ed.) D M Ginsberg (Singapore: World Scientific Publ.) Vol. II p. 3

Kresin V Z, Wolf S A and Morawitz H A 1993 Mechanism of conventional and high temperature superconductivity (New York: Oxford Univ. Press)
Leggett A 1999 Phys. Rev. Lett. 83392

Naumov V N, Frolova G I, Amitin E B, Fedonov V E and Samoilov P P 1996 Physica C262 143

Tarascan J M, Mc Kinnon W R, Greence L H, Hull G W and Vogel E M 1987 Phys. Rev. B36 226

Wu M K et al 1987 Phys. Rev. Lett. 58908 